\title{
INTERMEDIATE VALUES AND INVERSE FUNCTIONS ON NON-ARCHIMEDEAN FIELDS
}

\author{
KHODR SHAMSEDDINE and MARTIN BERZ
}

Received 27 April 2001 and in revised form 20 September 2001

\begin{abstract}
Continuity or even differentiability of a function on a closed interval of a non-Archimedean field are not sufficient for the function to assume all the intermediate values, a maximum, a minimum, or a unique primitive function on the interval. These problems are due to the total disconnectedness of the field in the order topology. In this paper, we show that differentiability (in the topological sense), together with some additional mild conditions, is indeed sufficient to guarantee that the function assumes all intermediate values and has a differentiable inverse function.
\end{abstract}

2000 Mathematics Subject Classification: 26E30, 12J25, 11D88.

1. Introduction. Let $K$ be a totally ordered non-Archimedean field extension of $\mathbb{R}$. We introduce the following terminology.

Definition $1.1(\sim, \approx, \ll, H, \lambda)$. For $x, y \in K, x \sim y$ if there exist $n, m \in \mathbb{N}$ such that $n|x|>|y|$ and $m|y|>|x|$; for nonnegative $x, y \in K, x$ is infinitely smaller than $y$ and write $x \ll y$ if $n x<y$ for all $n \in \mathbb{N}$; and $x$ is infinitely small if $x \ll 1$ and $x$ is finite if $x \sim 1$. Finally, $x$ is approximately equal to $y$ and write $x \approx y$ if $x \sim y$ and $|x-y| \ll|x|$. We also set $\lambda(x)=[x]$, the class of $x$ under the equivalence relation $\sim$.

The set $H$ of equivalence classes under the relation $\sim$, which we call magnitudes, is naturally endowed with an addition via $[x]+[y]=[x \cdot y]$, and an order via $[x]<[y]$ if $|y| \ll|x|$ (or $|x| \gg|y|$ ), both of which are readily checked to be well defined. It follows that $(H,+,<)$ is a totally ordered group, often referred to as the Hahn group or skeleton group, whose neutral element is the class of 1 . The projection $\lambda$ from $K$ to $H$ satisfies $\lambda(x \cdot y)=\lambda(x)+\lambda(y)$ and is a valuation.

The theorem of Hahn [5] provides a complete classification of any non-Archimedean extensions $K$ of $\mathbb{R}$ in terms of their skeleton group $H$. In fact, invoking the axiom of choice, it is shown that the elements of $K$ can be written as formal power series over the group $H$ with real coefficients, and the set of appearing "exponents" forms a wellordered subset of $H$. The coefficient of the $q$ th power in the Hahn representation of a given $x$ is denoted by $x[q]$, and the number $d$ is defined by $d[1]=1$ and $d[q]=0$ for $q \neq 1$. It is easy to check that $0<d^{q} \ll 1$ if and only if $q>0$, and $d^{q} \gg 1$ if and only if $q<0$; moreover, $x \approx x[\lambda(x)] d^{\lambda(x)}$ for all $x \neq 0$.

From general properties of formal power series fields [9, 11], it follows that if $H$ is divisible then $K$ is real-closed. For a general overview of the algebraic properties of formal power series fields, we refer to the comprehensive overview by Ribenboim [12] 
and Fuchs [4]; and for an overview of the related valuation theory, Krull [6]. A thorough and complete treatment of ordered structures can also be found in [10].

Throughout, $\mathcal{N}$ denotes any totally ordered non-Archimedean field extension of $\mathbb{R}$ that is complete in the order topology and whose skeleton group is Archimedean, that is, a subgroup of $\mathbb{R}$. The smallest such field is the field of the formal Laurent series whose skeleton group is $\mathbb{Z}$; and the smallest such field that is also real-closed is the field $\mathscr{R}$, first introduced by Levi-Civita [7, 8]. In this case $H=\mathbb{Q}$; and for any element $x \in \mathscr{R}$, the set of exponents in the Hahn representation of $x$ is a left-finite subset of $\mathbb{Q}$, that is, below any rational bound $r$ there are only finitely many exponents. For a detailed study of the Levi-Civita field $\mathscr{R}$, we refer the reader to [1, 2, 3, 13, 14, 15].

In this paper, we derive conditions under which a differentiable function assumes all intermediate values on a closed interval and has a differentiable inverse function. Previous versions of the intermediate value theorem were proved for the case of finite domain and range, and they were based on stronger smoothness criteria, namely equidifferentiability [3] and double derivative differentiability [1]. For the important class of locally analytic functions studied in detail in [13], we prove an intermediate value theorem (as well as a maximum theorem and a mean value theorem) without any requirements on the magnitude of the first derivative or the restriction of scaling into finite domains.

2. Review of continuity and differentiability. Like in any other metric space, continuity and differentiability at a point or on a domain of $\mathcal{N}$ are preserved under addition, multiplication, and composition of functions. We also have the following useful result.

Proposition 2.1. Let $D \subset \mathcal{N}$ be open, and let $f: D \rightarrow \mathcal{N}$ be differentiable on $D$ and have a local extremum (maximum or minimum) at $x_{0} \in D$. Then $f^{\prime}\left(x_{0}\right)=0$.

Proof. Suppose not, then $\left|f^{\prime}\left(x_{0}\right)\right|>0$. Since $D$ is open and since $f$ is differentiable at $x_{0}$, there exists $\delta>0$ in $\mathcal{N}$ such that $\left(x_{0}-\delta, x_{0}+\delta\right) \subset D$ and $\mid\left(f(x)-f\left(x_{0}\right)\right) /(x-$ $\left.x_{0}\right)-f^{\prime}\left(x_{0}\right)|<| f^{\prime}\left(x_{0}\right) \mid$ for all $x \neq x_{0}$ in $\left(x_{0}-\delta, x_{0}+\delta\right)$; which entails that $(f(x)-$ $\left.f\left(x_{0}\right)\right) /\left(x-x_{0}\right)$ has the same sign for all $x \neq x_{0}$ in $\left(x_{0}-\delta, x_{0}+\delta\right)$; and this contradicts the fact that $f$ has a local extremum at $x_{0}$.

However, contrary to the real case, the following examples show that continuity or differentiability of a function on a closed interval of $\mathcal{N}$ are not always sufficient for the function to assume all intermediate values, extrema, or even be bounded.

EXAMPLE 2.2. Let $f:[0,1] \rightarrow \mathcal{N}$ be given by

$$
f(x)= \begin{cases}d^{-1} & \text { if } 0 \leq x<d, \\ d^{-1 / \lambda(x)} & \text { if } d \leq x \ll 1, \\ 1 & \text { if } x \sim 1\end{cases}
$$

Then $f$ is continuous on $[0,1]$; but for $d \leq x \ll 1, f(x)$ grows without bound. 
EXAMPLE 2.3. Let $f:[0,1] \rightarrow \mathcal{N}$ be given by

$$
f(x)= \begin{cases}1 & \text { if } x \sim 1 \\ 0 & \text { if } 0 \leq x \ll 1\end{cases}
$$

Then $f$ is differentiable on $[0,1]$, with derivative $f^{\prime}(x)=0$ for all $x$. However, $f$ does not assume the intermediate value $d$ on $[0,1]$. Moreover, although $f^{\prime}(x)$ is identically null, $f$ is not constant on $[0,1]$.

EXAMPLE 2.4. Let $f:[-1,1] \rightarrow \mathcal{N}$ be given by $f(x)=x-x[0]$. Then $f$ is continuous on $[-1,1]$. However, $f$ assumes neither a maximum nor a minimum on $[-1,1]$. The set $f([-1,1])$ is bounded above by any positive real number and below by any negative real number; but it has neither a least upper bound nor a greatest lower bound.

In the following section, we study a large class of differentiable functions and show that they assume all intermediate values on a closed interval and a differentiable inverse function.

3. Intermediate value theorem and inverse function theorem. First, we state the following result which will be used in the proof of Theorem 3.17, and we refer the reader to [3] for its proof.

THEOREM 3.1 (fixed point theorem). Let $q_{M} \in \mathbb{R}$ be given. Define $M \subset \mathcal{N}$ to be the set of all elements $x$ of $\mathcal{N}$ such that $\lambda(x) \geq q_{M}$. Let $f: M \rightarrow \mathcal{N}$ satisfy $f(M) \subset M$. Suppose that there exists $k>0$ in $\mathbb{R}$ such that for all $x_{1}, x_{2} \in M, \lambda\left(f\left(x_{2}\right)-f\left(x_{1}\right)\right) \geq k+\lambda\left(x_{2}-x_{1}\right)$. Then there exists a unique solution $x \in M$ of the fixed point equation $x=f(x)$.

Definition 3.2. Let $a<b$ be given in $\mathcal{N}$, and let $f:[a, b] \rightarrow \mathcal{N}$ be differentiable. Then $f$ is an IVT-function on $[a, b]$ if there exists $n \in \mathbb{N}$ such that

$$
\begin{aligned}
\frac{f(y)-f(x)}{y-x} & \sim \frac{f(b)-f(a)}{b-a}, \\
\frac{\left|f(y)-f(x)-f^{\prime}(x)(y-x)\right|}{(y-x)^{2}} & \leq n \cdot \frac{\left|f(b)-f(a)-f^{\prime}(a)(b-a)\right|}{(b-a)^{2}}
\end{aligned}
$$

for all $y \neq x$ in $[a, b]$.

The acronym IVT in Definition 3.2 stands for intermediate value theorem. As we will see in Theorem 3.17, an IVT-function on a closed interval $[a, b]$ assumes every intermediate value between $f(a)$ and $f(b)$; hence the name.

It follows immediately from Definition 3.2 that

$$
f^{\prime}(x) \sim \frac{f(b)-f(a)}{b-a} \quad \forall x \in[a, b] .
$$

REMARK 3.3. It is easy to check that the property introduced in Definition 3.2 is preserved under scaling and translation. That is, if $f:[a, b] \rightarrow \mathcal{N}$ is an IVT-function on $[a, b]$, then for all $c_{1} \neq 0, c_{2}, c_{3}, c_{4}$ in $\mathcal{N}$, the function

$$
g:\left[\frac{a-c_{2}}{c_{1}}, \frac{b-c_{2}}{c_{1}}\right] \rightarrow \mathcal{N}
$$


given by $g(x)=c_{3} f\left(c_{1} x+c_{2}\right)+c_{4}$, is an IVT-function on $\left[\left(a-c_{2}\right) / c_{1},\left(b-c_{2}\right) / c_{1}\right]$. In fact, replacing $f$ by $g, a$ by $\left(a-c_{2}\right) / c_{1}$, and $b$ by $\left(b-c_{2}\right) / c_{1}$ yields the same factor $c_{1} c_{3}$ on both sides of (3.1), and the same factor $c_{1}^{2} c_{3}$ on both sides of (3.2).

We show in Theorem 3.17 that if $f$ is an IVT-function on $[a, b]$ then $f$ assumes every intermediate value between $f(a)$ and $f(b)$ and has a differentiable inverse function. The two conditions in Definition 3.2 may seem strange, but the first condition means that the function is either constant or one-to-one with slope of uniform magnitude; when restricted to $\mathbb{R}$, the uniformity of the magnitude is automatic. Also, when restricted to $\mathbb{R}$, the second condition means merely that the difference quotient is bounded. Moreover, the following two examples show that one of the two conditions alone will not be sufficient.

EXAMPLE 3.4. Let $f:[0,1] \rightarrow \mathcal{N}$ be given by

$$
f(x)= \begin{cases}3 x[0]+(x-x[0])+(x-x[0])^{2} & \text { if } x[0] \text { is rational } \\ 2 x[0]+(x-x[0])+(x-x[0])^{2} & \text { if } x[0] \text { is irrational }\end{cases}
$$

Then $f$ is differentiable on $[0,1]$ with derivative $f^{\prime}(x)=1$ for all $x$. Clearly, $f$ does not assume the value $3 \pi / 4$ which lies between $f(0)=0$ and $f(1)=3$. Here, (3.1) is satisfied since

$$
\frac{f(y)-f(x)}{y-x} \sim \frac{f(1)-f(0)}{1-0}=3 \quad \forall y \neq x \text { in }[0,1]
$$

but (3.2) does not hold. In this example, we even have that

$$
\frac{f(y)-f(x)-f^{\prime}(x)(y-x)}{(y-x)^{2}} \sim \frac{f(1)-f(0)-f^{\prime}(0)(1-0)}{(1-0)^{2}} \quad \forall y \neq x \text { in }[0,1] .
$$

EXAMPLE 3.5. Let $f:[0,1] \rightarrow \mathcal{N}$ be given by

$$
f(x)= \begin{cases}0 & \text { if } 0 \leq x \ll 1 \\ x & \text { if } x \sim 1\end{cases}
$$

Then $f$ is differentiable on $[0,1]$ with derivative $f^{\prime}(x)=0$ if $0 \leq x \ll 1$ and $f^{\prime}(x)=1$ if $x \sim 1$. Clearly, $f$ does not assume the value $d$ which lies between $f(0)=0$ and $f(1)=1$. Here (3.2) is satisfied since

$$
\begin{aligned}
& \frac{\left|f(y)-f(x)-f^{\prime}(x)(y-x)\right|}{(y-x)^{2}} \\
& \quad<3\left|f(1)-f(0)-f^{\prime}(0)\right|=3 \quad \forall y \neq x \text { in }[0,1] ;
\end{aligned}
$$


but (3.1) does not hold since

$$
\frac{f(y)-f(x)}{y-x}=0 \nmid 1=f(1)-f(0) \quad \text { for infinitely small } x, y \in[0,1] \text {. }
$$

REMARK 3.6. Examples of IVT-functions on $[0,1]$ are polynomials and power series with real coefficients and with finite first derivative throughout the interval, functions that are equidifferentiable on [0,1] as in [3], and functions that are twice differentiable on $[0,1]$ in the derivative sense of [1] with finite first and second derivatives. Thus, the intermediate value theorem we prove below is a generalization of the previous two versions in [1, 3]; moreover, it will apply for functions on an interval of any size and not just intervals of finite length.

LEMmA 3.7. Let $a<b$ be given in $\mathcal{N}$, and let $f:[a, b] \rightarrow \mathcal{N}$ be an IVT-function. Then there exists $m \in \mathbb{N}$ such that

$$
\left|\frac{f(y)-f(x)}{y-x}-f^{\prime}(x)\right| \leq m \frac{|f(b)-f(a)|}{(b-a)^{2}}|y-x| \quad \forall y \neq x \text { in }[a, b] .
$$

Proof. Let $n \in \mathbb{N}$ be as in (3.2). Using (3.3), we have that $f^{\prime}(a) \sim(f(b)-f(a))$ / $(b-a)$; and hence there exists $k \in \mathbb{N}$ such that $\left|f^{\prime}(a)\right| \leq k \cdot|f(b)-f(a)| /(b-a)$. Thus,

$$
\begin{aligned}
\frac{\left|f(b)-f(a)-f^{\prime}(a)(b-a)\right|}{b-a} & \leq \frac{|f(b)-f(a)|}{b-a}+\left|f^{\prime}(a)\right| \\
& \leq(1+k) \frac{|f(b)-f(a)|}{b-a} .
\end{aligned}
$$

Hence

$$
\begin{aligned}
\left|\frac{f(y)-f(x)}{y-x}-f^{\prime}(x)\right| & \leq n \frac{\left|f(b)-f(a)-f^{\prime}(a)(b-a)\right|}{(b-a)^{2}}|y-x| \\
& \leq n(1+k) \frac{|f(b)-f(a)|}{(b-a)^{2}}|y-x|
\end{aligned}
$$

for all $y \neq x$ in $[a, b]$.

COROLLARY 3.8 (remainder formula). Let $a<b$ be given in $\mathcal{N}$, and let $f:[a, b] \rightarrow \mathcal{N}$ be an IVT-function. Then, for all $x, y \in[a, b]$,

$$
f(y)=f(x)+f^{\prime}(x)(y-x)+r(x, y)(y-x)^{2},
$$

with

$$
\lambda(r(x, y)) \geq \lambda\left(\frac{f(b)-f(a)}{(b-a)^{2}}\right)
$$


Proof. For $x, y \in[a, b]$, let

$$
r(x, y)= \begin{cases}\frac{f(y)-f(x)-f^{\prime}(x)(y-x)}{(y-x)^{2}} & \text { if } y \neq x \\ 0 & \text { if } y=x .\end{cases}
$$

Then $f(y)=f(x)+f^{\prime}(x)(y-x)+r(x, y)(y-x)^{2}$ for all $x, y \in[a, b]$. Moreover, using Lemma 3.7, we obtain that $\lambda(r(x, y)) \geq \lambda\left((f(b)-f(a)) /(b-a)^{2}\right)$, as claimed.

REMARK 3.9. The remainder formula here resembles that obtained in [1] as a result of the derivative differentiability, but we have the extra condition that $\lambda(r(x, y)) \geq$ $\lambda\left((f(b)-f(a)) /(b-a)^{2}\right)$ which is useful for proving Theorem 3.17.

LEMMA 3.10. Let $a<b$ be given in $\mathcal{N}$, and let $f:[a, b] \rightarrow \mathcal{N}$ be an IVT-function. Then $f$ is continuously differentiable on $[a, b]$.

Proof. Let $m \in \mathbb{N}$ be as in Lemma 3.7, and let $x \neq y$ in $[a, b]$ be given. Then

$$
\begin{aligned}
\left|f^{\prime}(y)-f^{\prime}(x)\right| & \leq\left|\frac{f(y)-f(x)}{y-x}-f^{\prime}(y)\right|+\left|\frac{f(y)-f(x)}{y-x}-f^{\prime}(x)\right| \\
& \leq 2 m \frac{|f(b)-f(a)|}{(b-a)^{2}}|y-x| .
\end{aligned}
$$

Hence $f^{\prime}$ is continuous on $[a, b]$.

COROLLARY 3.11. Let $a<b$ be given in $\mathcal{N}$, and let $f:[a, b] \rightarrow \mathcal{N}$ be an IVT-function. Then, for all $x, y \in[a, b]$,

$$
\lambda\left(f^{\prime}(y)-f^{\prime}(x)\right) \geq \lambda\left(\frac{f(b)-f(a)}{b-a}\right)+\lambda\left(\frac{y-x}{b-a}\right) .
$$

LEMMA 3.12. Let $a<b$ be given in $\mathcal{N}$, and let $f:[a, b] \rightarrow \mathcal{N}$ be an IVT-function. If $f(a)=f(b)$, then $f$ is constant on $[a, b]$.

Proof. Let $x \in(a, b]$ be given. Then $(f(x)-f(a)) /(x-a) \sim(f(b)-f(a)) /$ $(b-a)=0$, which entails that $f(x)=f(a)$.

LEMmA 3.13. Let $a<b$ be given in $\mathcal{N}$, let $f:[a, b] \rightarrow \mathcal{N}$ be a nonconstant IVTfunction, and let $g:[0,1] \rightarrow \mathcal{N}$ be given by

$$
g(x)=\frac{f((b-a) x+a)-f(a)}{f(b)-f(a)} .
$$

Then $g$ is an IVT-function on $[0,1]$, with $\lambda(g(x))=\lambda(x) \geq 0$ and $\lambda\left(g^{\prime}(x)\right)=0$ for all $x \in[0,1]$. 
Proof. The proof that $g$ is an IVT-function on $[0,1]$ follows from Remark 3.3. Now let $x \in[0,1]$ be given. Then,

$$
\begin{aligned}
\lambda(g(x)) & =\lambda\left(\frac{f((b-a) x+a)-f(a)}{(b-a) x} \frac{(b-a) x}{f(b)-f(a)}\right) \\
& =\lambda\left(\frac{f((b-a) x+a)-f(a)}{((b-a) x+a)-a}\right)+\lambda\left(\frac{b-a}{f(b)-f(a)}\right)+\lambda(x) \\
& =\lambda\left(\frac{f(b)-f(a)}{b-a}\right)+\lambda\left(\frac{b-a}{f(b)-f(a)}\right)+\lambda(x)=\lambda(x) \geq 0 .
\end{aligned}
$$

Moreover, $g^{\prime}(x)=(b-a) /(f(b)-f(a)) \cdot f^{\prime}((b-a) x+a) \sim 1$, using (3.3).

The following result follows immediately from Lemma 3.13 and Corollary 3.11.

COROLlary 3.14. Let $a<b$ be given in $\mathcal{N}$, let $f:[a, b] \rightarrow \mathcal{N}$ be a nonconstant IVT-function, and let $g:[0,1] \rightarrow \mathcal{N}$ be as in Lemma 3.13. Then, for all $x, y \in[0,1]$, $\lambda\left(g^{\prime}(y)-g^{\prime}(x)\right) \geq \lambda(y-x)$.

LEMMA 3.15. Let $a<b$ be given in $\mathcal{N}$, let $f:[a, b] \rightarrow \mathcal{N}$ be a nonconstant IVTfunction, and let $g:[0,1] \rightarrow \mathcal{N}$ be as in Lemma 3.13. Let $g_{R}:[0,1] \cap \mathbb{R} \rightarrow \mathbb{R}$ be given by $g_{R}(X)=g(X)[0]$. Then $g_{R}$ is continuously differentiable on $[0,1] \cap \mathbb{R}$ (in the real sense), with derivative $\left(g_{R}\right)^{\prime}(X)=g^{\prime}(X)[0] \neq 0$ for all $X \in[0,1] \cap \mathbb{R}$.

Proof. Since $g$ is an IVT-function on $[0,1]$ by Lemma 3.13, there exists $m \in \mathbb{N}$ by Lemma 3.7 such that

$$
\left|\frac{g(y)-g(x)}{y-x}-g^{\prime}(x)\right| \leq m|y-x| \quad \forall y \neq x \text { in }[0,1] .
$$

Now, let $X \in[0,1] \cap \mathbb{R}$ be given. Then

$$
\left|\frac{g(Y)-g(X)}{Y-X}-g^{\prime}(X)\right| \leq m|Y-X| \quad \forall Y \neq X \text { in }[0,1] \cap \mathbb{R} .
$$

Thus, for all $Y \neq X$ in $[0,1] \cap \mathbb{R}$, we have that

$$
\left|\frac{g_{R}(Y)-g_{R}(X)}{Y-X}-g^{\prime}(X)[0]\right|=\left|\left(\frac{g(Y)-g(X)}{Y-X}-g^{\prime}(X)\right)[0]\right| \leq 2 m|Y-X|,
$$

which entails that $g_{R}$ is differentiable (in the real sense) at $X$ with derivative $\left(g_{R}\right)^{\prime}(X)=$ $g^{\prime}(X)[0] \neq 0$, since $\lambda\left(g^{\prime}(X)\right)=0$ by Lemma 3.13 .

Next, we show that $\left(g_{R}\right)^{\prime}$ is continuous on $[0,1] \cap \mathbb{R}$. As in the proof of Lemma 3.10, we have that $\left|g^{\prime}(y)-g^{\prime}(x)\right| \leq 2 m|y-x|$ for all $x, y \in[0,1]$. In particular, $\mid g^{\prime}(Y)-$ $g^{\prime}(X)|\leq 2 m| Y-X \mid$ for all $X, Y \in[0,1] \cap \mathbb{R}$. It follows that

$$
\left|\left(g_{R}\right)^{\prime}(Y)-\left(g_{R}\right)^{\prime}(X)\right|=\left|g^{\prime}(Y)[0]-g^{\prime}(X)[0]\right| \leq 3 m|Y-X|
$$

for all $X, Y \in[0,1] \cap \mathbb{R}$, which entails that $\left(g_{R}\right)^{\prime}$ is (uniformly) continuous on $[0,1] \cap \mathbb{R}$. Thus, $g_{R}$ is continuously differentiable on $[0,1] \cap \mathbb{R}$. 
LEMMA 3.16. Let $a<b$ be given in $\mathcal{N}$, and let $f:[a, b] \rightarrow \mathcal{N}$ be a nonconstant IVTfunction. Then $f$ is strictly monotone on $[a, b]$.

Proof. Let $g:[0,1] \rightarrow \mathcal{N}$ be as in Lemma 3.13. We show that $g$ is strictly increasing on $[0,1]$. Let $g_{R}$ be as in Lemma 3.15. Then $g_{R}$ is continuously differentiable on $[0,1] \cap \mathbb{R}$ and $\left(g_{R}\right)^{\prime}(X) \neq 0$ for all $X \in[0,1] \cap \mathbb{R}$. Thus, $g_{R}$ is strictly monotone on $[0,1] \cap \mathbb{R}$. Since $g_{R}(0)=0<1=g_{R}(1)$, we obtain that $g_{R}$ is strictly increasing on $[0,1] \cap \mathbb{R}$. Now let $x, y \in[0,1]$ be such that $x<y$, and let $X=x[0]$ and $Y=y[0]$. As a first case, assume that $X<Y$; then $g_{R}(X)<g_{R}(Y)$. Hence

$$
\begin{aligned}
g(y)-g(x)= & \left(g_{R}(Y)-g_{R}(X)\right)+(g(y)-g(Y))+\left(g(Y)-g_{R}(Y)\right) \\
& +\left(g_{R}(X)-g(X)\right)+(g(X)-g(x)),
\end{aligned}
$$

where the first term is positive and real. By Corollary 3.8, we have that $g(y)-g(Y)=$ $g^{\prime}(Y)(y-Y)+r(Y, y)(y-Y)^{2}$, where $\lambda\left(g^{\prime}(Y)\right)=0, \lambda(y-Y)>0$, and $\lambda(r(Y, y)) \geq 0$. Hence $|g(y)-g(Y)|$ is infinitely small. Similarly, $|g(X)-g(x)|$ is infinitely small. Since $\lambda(g(Y)) \geq 0$ and $g_{R}(Y)=g(Y)[0]$, we obtain that $\left|g(Y)-g_{R}(Y)\right|$ is infinitely small. Similarly, $\left|g_{R}(X)-g(X)\right|$ is infinitely small. So $g(y)-g(x) \approx g_{R}(Y)-g_{R}(X)>0$; and hence $g(x)<g(y)$.

As a second case, assume that $X=Y$. Then $y-x \ll 1$, and hence

$$
g(y)-g(x)=g^{\prime}(x)(y-x)+r(x, y)(y-x)^{2} \approx g^{\prime}(x)(y-x)
$$

since $|r(x, y)|$ is at most finite and hence

$$
\begin{aligned}
\lambda\left(r(x, y)(y-x)^{2}\right) & =\lambda(r(x, y))+2 \lambda(y-x) \geq 2 \lambda(y-x)>\lambda(y-x) \\
& =\lambda\left(g^{\prime}(x)\right)+\lambda(y-x) \\
& =\lambda\left(g^{\prime}(x)(y-x)\right) .
\end{aligned}
$$

By Corollary 3.14, we have that $\lambda\left(g^{\prime}(x)-g^{\prime}(X)\right) \geq \lambda(x-X)>0$. Since $g^{\prime}(x) \sim 1$, since $g^{\prime}(X) \sim 1$ and since $\left|g^{\prime}(x)-g^{\prime}(X)\right| \ll 1$, we obtain that

$$
g^{\prime}(x) \approx g^{\prime}(X) \approx\left(g_{R}\right)^{\prime}(X)>0 .
$$

From (3.26) and (3.28), we obtain that $g(y)-g(x)>0$. Thus, $g(x)<g(y)$ for all $x<y$ in $[0,1]$; and hence $g$ is strictly increasing on $[0,1]$. Since

$$
f(x)=(f(b)-f(a)) g\left(\frac{x-a}{b-a}\right)+f(a) \quad \forall x \in[a, b]
$$

and since $g$ is strictly increasing on $[0,1]$, we obtain that $f$ is strictly increasing on $[a, b]$ if $f(a)<f(b)$, and $f$ is strictly decreasing on $[a, b]$ if $f(a)>f(b)$.

THEOREM 3.17 (intermediate value theorem). Let $a<b$ be given in $\mathcal{N}$, and let $f$ : $[a, b] \rightarrow \mathcal{N}$ be an IVT-function. Then $f$ assumes every intermediate value between $f(a)$ and $f(b)$.

Proof. If $f(a)=f(b)$, then $f$ is constant on $[a, b]$ by Lemma 3.12, and there is nothing to prove. So we may assume that $f(a) \neq f(b)$. Let $g:[0,1] \rightarrow \mathcal{N}$ be as in 
Lemma 3.13. For all $x \in[a, b]$, we have that

$$
f(x)=(f(b)-f(a)) g\left(\frac{x-a}{b-a}\right)+f(a)=l_{2} \circ g \circ l_{1}(x),
$$

where $l_{1}$ and $l_{2}$ are linear functions. Hence it suffices to show that $g$ assumes every intermediate value between $g(0)=0$ and $g(1)=1$.

Let $g_{R}$ be as in Lemma 3.15, let $S \in(0,1)$ be given, and let $S_{R}=S[0]$. Then $S_{R} \in$ $[0,1] \cap \mathbb{R}$. Since $g_{R}$ is continuous on $[0,1] \cap \mathbb{R}$ by Lemma 3.15 , there exists $X \in[0,1] \cap \mathbb{R}$ such that $g_{R}(X)=S_{R}$. If $g(X)=S$ then the claim is proved; so we may assume that $g(X) \neq S$. Thus, $|S-g(X)| \leq\left|S-S_{R}\right|+\left|g_{R}(X)-g(X)\right|$ is infinitely small.

Now we proceed to find $x$ such that $0<|x| \ll 1, X+x \in[0,1]$, and $g(X+x)=S$. Since $g$ is differentiable on $[0,1]$, we have, using Corollary 3.8, that

$$
S=g(X+x)=g(X)+g^{\prime}(X) x+r(X, X+x) x^{2},
$$

where $|r(X, X+x)|$ is at most finite.

Transforming (3.31) into a fixed point problem yields

$$
x=\frac{s}{g^{\prime}(X)}-\frac{r(X, X+x)}{g^{\prime}(X)} x^{2}=h(x),
$$

where $s=S-g(X)$, and $|s|$ is infinitely small. Let $M=\{z \in \mathcal{N}: \lambda(z) \geq \lambda(s)\}$ and let $x \in M$ be given. Since $|r(X, X+x)|$ is at most finite and since $g^{\prime}(X) \sim 1$, we have that

$$
\lambda\left(\frac{r(X, X+x)}{g^{\prime}(X)} x^{2}\right) \geq 2 \lambda(x)>\lambda(x) \geq \lambda(s)=\lambda\left(\frac{s}{g^{\prime}(X)}\right) .
$$

Thus, $h(x) \approx s / g^{\prime}(X)$; and hence $\lambda(h(x))=\lambda(s)$ for all $x \in M$. Hence $h(M) \subset M$. Now let $x_{1} \neq x_{2}$ be given in $M$. Then

$$
\begin{aligned}
\left|h\left(x_{1}\right)-h\left(x_{2}\right)\right| & =\left|\frac{r\left(X, X+x_{2}\right) x_{2}^{2}-r\left(X, X+x_{1}\right) x_{1}^{2}}{g^{\prime}(X)}\right| \\
& =\left|\frac{g\left(X+x_{2}\right)-g\left(X+x_{1}\right)}{g^{\prime}(X)}+x_{1}-x_{2}\right| .
\end{aligned}
$$

But $g\left(X+x_{2}\right)=g\left(X+x_{1}\right)+g^{\prime}\left(X+x_{1}\right)\left(x_{2}-x_{1}\right)+r\left(X+x_{1}, X+x_{2}\right)\left(x_{2}-x_{1}\right)^{2}$, where $\left|r\left(X+x_{1}, X+x_{2}\right)\right|$ is at most finite. Thus,

$$
\begin{aligned}
& \left|h\left(x_{1}\right)-h\left(x_{2}\right)\right| \\
& \quad=\left|\frac{g^{\prime}\left(X+x_{1}\right)\left(x_{2}-x_{1}\right)+r\left(X+x_{1}, X+x_{2}\right)\left(x_{2}-x_{1}\right)^{2}}{g^{\prime}(X)}+x_{1}-x_{2}\right| \\
& \quad=\left|\frac{g^{\prime}\left(X+x_{1}\right)-g^{\prime}(X)}{g^{\prime}(X)}\left(x_{2}-x_{1}\right)+\frac{r\left(X+x_{1}, X+x_{2}\right)}{g^{\prime}(X)}\left(x_{2}-x_{1}\right)^{2}\right| \\
& \quad \leq\left|x_{1}-x_{2}\right|\left(\frac{\left|g^{\prime}\left(X+x_{1}\right)-g^{\prime}(X)\right|}{g^{\prime}(X)}+\frac{\left|r\left(X+x_{1}, X+x_{2}\right)\right|}{g^{\prime}(X)}\left|x_{1}-x_{2}\right|\right) .
\end{aligned}
$$


Using Corollary 3.14 and the fact that $g^{\prime}(X) \sim 1$, we have that

$$
\lambda\left(\frac{\left|g^{\prime}\left(X+x_{1}\right)-g^{\prime}(X)\right|}{g^{\prime}(X)}\right)=\lambda\left(g^{\prime}\left(X+x_{1}\right)-g^{\prime}(X)\right) \geq \lambda\left(x_{1}\right) \geq \lambda(s)>\frac{\lambda(s)}{2} .
$$

Also

$$
\lambda\left(\frac{\left|r\left(X+x_{1}, X+x_{2}\right)\right|}{g^{\prime}(X)}\left|x_{1}-x_{2}\right|\right) \geq \lambda\left(x_{1}-x_{2}\right) \geq \min \left\{\lambda\left(x_{1}\right), \lambda\left(x_{2}\right)\right\}>\frac{\lambda(s)}{2} .
$$

Hence $\lambda\left(h\left(x_{1}\right)-h\left(x_{2}\right)\right)>\lambda(s) / 2+\lambda\left(x_{1}-x_{2}\right)$, where $\lambda(s)>0$. So $h$ and $M$ satisfy the requirements of Theorem 3.1, and hence $h$ has a fixed point $x$ in $M$.

Finally, we show that $X+x \in(0,1)$. First assume that $X=0$; then $S>0=g(X)$ and hence $s=S-g(X)>0$. Since $g^{\prime}(0) \approx\left(g_{R}\right)^{\prime}(0)>0$, we obtain that $X+x=x \approx$ $s / g^{\prime}(0)>0$. Moreover, $x \ll 1$; hence $X+x=x \in(0,1)$. Now assume that $X=1$, then $S<1=g(1)$ and hence $s<0$. It follows that $x \approx s / g^{\prime}(1)<0$ and hence $X+$ $x=1+x<1$. Since $|x| \ll 1$, we obtain that $X+x=1+x \in(0,1)$. Finally assume that $0<X<1$; then $X$ is finitely away from 0 and 1 . Since $|x| \ll 1$, we obtain that $X+x \in(0,1)$.

Using Lemma 3.16 and Theorem 3.17, we readily obtain the following two results.

COROLlary 3.18. Let $a<b$ be given in $\mathcal{N}$, and let $f:[a, b] \rightarrow \mathcal{N}$ be a nonconstant IVT-function. Let $m=\min \{f(a), f(b)\}$ and $M=\max \{f(a), f(b)\}$. Then $f([a, b])=$ $[m, M]$.

THEOREM 3.19 (closed mapping theorem). Let $a, b, f, m$, and $M$ be as in Corollary 3.18. Then for all $a_{1}<b_{1}$ in $[a, b]$, there exist $m_{1}<M_{1}$ in $[m, M]$ such that $f\left(\left[a_{1}, b_{1}\right]\right)=$ $\left[m_{1}, M_{1}\right]$. Conversely, for all $m_{1}<M_{1}$ in $[m, M]$, there exist $a_{1}<b_{1}$ in $[a, b]$ such that $f\left(\left[a_{1}, b_{1}\right]\right)=\left[m_{1}, M_{1}\right]$.

We note here that even though the conditions in Definition 3.2 depend on the end points $a$ and $b$, the function $f$ assumes all intermediate values between $f\left(a_{1}\right)$ and $f\left(b_{1}\right)$ for any subinterval $\left[a_{1}, b_{1}\right]$ of $[a, b]$.

THEOREM 3.20 (inverse function theorem). Let $a<b$ be given in $\mathcal{N}$, and let $f$ : $[a, b] \rightarrow \mathcal{N}$ be a nonconstant IVT-function. Let $m=\min \{f(a), f(b)\}$ and $M=$ $\max \{f(a), f(b)\}$. Then the inverse function $f^{-1}:[m, M] \rightarrow[a, b]$ exists and is differentiable; moreover,

$$
\left(f^{-1}\right)^{\prime}=\frac{1}{\left(f^{\prime} \circ f^{-1}\right)}
$$

Proof. The proof that $f^{-1}$ exists follows from Lemma 3.16. To show that $f^{-1}$ is differentiable on $[m, M]$, let $y_{0} \in[m, M]$ be given and let $x_{0}=f^{-1}\left(y_{0}\right)$. Let $\epsilon>0$ in $\mathcal{N}$ be given and let $\epsilon_{1} \in(0, \epsilon)$ be such that $\left|\left(f(x)-f\left(x_{0}\right)\right) /\left(x-x_{0}\right)-f^{\prime}\left(x_{0}\right)\right|<$ $\min \left\{\left|f^{\prime}\left(x_{0}\right)\right| / 2\right.$, $\left.\epsilon\left|f^{\prime}\left(x_{0}\right)\right|^{2} / 2\right\}$ for $x \in[a, b]$ satisfying $0<\left|x-x_{0}\right|<\epsilon_{1}$. It follows that $\mid f(x)-$ $f\left(x_{0}\right)|>| f^{\prime}\left(x_{0}\right)|| x-x_{0} \mid / 2$ when $x \in[a, b]$ and $0<\left|x-x_{0}\right|<\epsilon_{1}$. By Theorem 3.19, 
there exist $\delta_{1}, \delta_{2}>0$ such that $f\left([a, b] \cap\left[x_{0}-\epsilon_{1} / 2, x_{0}+\epsilon_{1} / 2\right]\right)=\left[y_{0}-\delta_{1}, y_{0}+\delta_{2}\right]$. Let $\delta=\min \left\{\delta_{1}, \delta_{2}\right\}$. Then $f^{-1}\left(\left(y_{0}-\delta, y_{0}+\delta\right)\right) \subset[a, b] \cap\left(x_{0}-\epsilon_{1}, x_{0}+\epsilon_{1}\right)$.

Now, let $y \in[m, M]$ be such that $0<\left|y-y_{0}\right|<\delta$. Then

$$
\begin{aligned}
\left|\frac{f^{-1}(y)-f^{-1}\left(y_{0}\right)}{y-y_{0}}-\frac{1}{f^{\prime}\left(x_{0}\right)}\right| & =\left|\frac{x-x_{0}}{f(x)-f\left(x_{0}\right)}-\frac{1}{f^{\prime}\left(x_{0}\right)}\right| \\
& =\frac{\left|x-x_{0}\right| \cdot\left|\left(f(x)-f\left(x_{0}\right)\right) /\left(x-x_{0}\right)-f^{\prime}\left(x_{0}\right)\right|}{\left|f^{\prime}\left(x_{0}\right)\right| \cdot\left|f(x)-f\left(x_{0}\right)\right|} \\
& <\frac{\left|x-x_{0}\right| \cdot \epsilon\left|f^{\prime}\left(x_{0}\right)\right|^{2} / 2}{\left|f^{\prime}\left(x_{0}\right)\right| \cdot\left|f(x)-f\left(x_{0}\right)\right|} \\
& <\frac{\left|x-x_{0}\right| \cdot \epsilon\left|f^{\prime}\left(x_{0}\right)\right|^{2} / 2}{\left|f^{\prime}\left(x_{0}\right)\right| \cdot\left|f^{\prime}\left(x_{0}\right)\right|\left|x-x_{0}\right| / 2} \\
& =\epsilon .
\end{aligned}
$$

Hence $f^{-1}$ is differentiable at $y_{0}$, and $\left(f^{-1}\right)^{\prime}\left(y_{0}\right)=1 / f^{\prime}\left(x_{0}\right)=1 / f^{\prime}\left(f^{-1}\left(y_{0}\right)\right)$.

ACKNOWLEDGments. This research was supported by an Alfred P. Sloan fellowship and by the Untied States Department of Energy, Grant \# DE-FG02-95ER40931.

\section{REFERENCES}

[1] M. Berz, Elements of analysis on non-Archimedean Levi-Civita fields, in preparation.

[2] _ Calculus and numerics on Levi-Civita fields, Computational Differentiation (M. Berz, C. Bischof, G. Corliss, and A. Griewank, eds.), SIAM, Pennsylvania, 1996, pp. 19-35.

[3] _ Analytical and computational methods for the Levi-Civita field, $p$-adic Functional Analysis (Ioannina, 2000), Lecture Notes in Pure and Applied Mathematics, vol. 222, Marcel Dekker, New York, 2001, pp. 21-34.

[4] L. Fuchs, Partially Ordered Algebraic Systems, Pergamon Press, Oxford, 1963.

[5] H. Hahn, Über die nichtarchimedischen Größensysteme, Wien, math.-naturw. Kl. Abt. iia 116 (1907), 601-655 (German).

[6] W. Krull, Allgemeine Bewertungstheorie, J. Reine Angew. Math. 167 (1932), 160-196 (German).

[7] T. Levi-Civita, Sugli infiniti ed infinitesimi attuali quali elementi analitici, Atti Ist. Veneto di Sc., Lett. ed Art., 7a 4 (1892), 1765-1815 (Italian).

[8]__ Sui numeri transfiniti, Rend. Acc. Lincei, 5a 7 (1898), 91-113 (Italian).

[9] S. MacLane, The universality of formal power series fields, Bull. Amer. Math. Soc. 45 (1939), 888-890.

[10] S. Prieß-Crampe, Angeordnete Strukturen. Gruppen, Körper, Projektive Ebenen [Ordered Structures. Groups, Fields, Projective Planes], Ergebnisse der Mathematik und ihrer Grenzgebiete, vol. 98, Springer-Verlag, Berlin, 1983 (German).

[11] F. J. Rayner, Algebraically closed fields analogous to fields of Puiseux series, J. London Math. Soc. (2) 8 (1974), 504-506.

[12] P. Ribenboim, Fields: algebraically closed and others, Manuscripta Math. 75 (1992), no. 2, $115-150$.

[13] K. Shamseddine and M. Berz, Analytical properties of power series on non-Archimedean fields, in preparation.

[14] _ Exception handling in derivative computation with non-Archimedean calculus, Computational Differentiation (M. Berz, C. Bischof, G. Corliss, and A. Griewank, eds.), SIAM, Pennsylvania, 1996, pp. 37-51. 
[15] C Convergence on the Levi-Civita field and study of power series, $p$-adic Functional Analysis (Ioannina, 2000), Lecture Notes in Pure and Applied Mathematics, vol. 222, Dekker, New York, 2001, pp. 283-299.

Khodr Shamseddine: Department of Mathematics, Michigan State University, East LANSING, MI 48824, USA

E-mail address: khodr@math.msu.edu

Martin Berz: Department of Physics and Astronomy, Michigan State University, EAST LANSING, MI 48824, USA

E-mail address: berz@msu. edu 


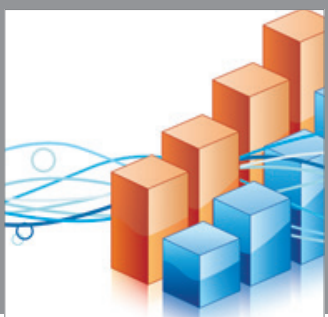

Advances in

Operations Research

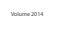

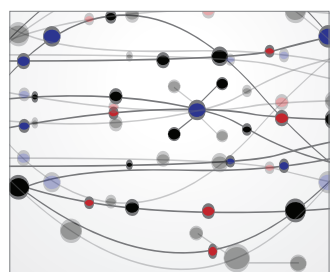

\section{The Scientific} World Journal
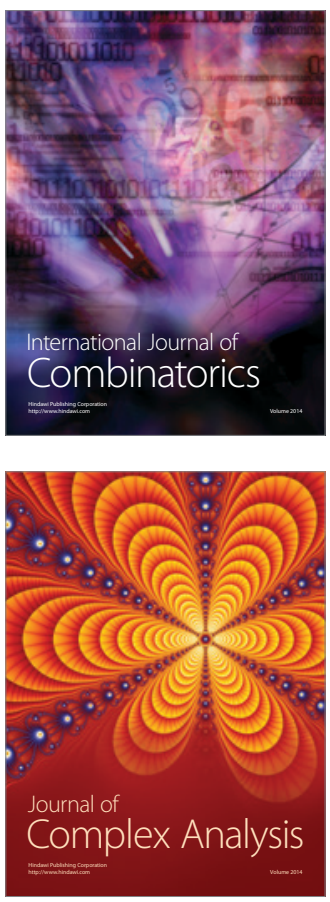

International Journal of

Mathematics and

Mathematical

Sciences
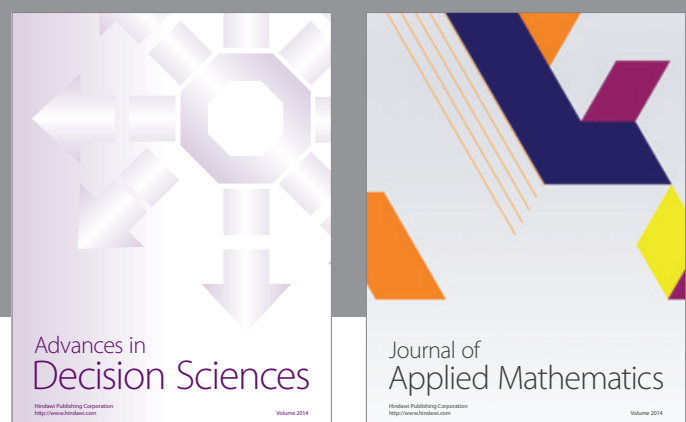

Journal of

Applied Mathematics
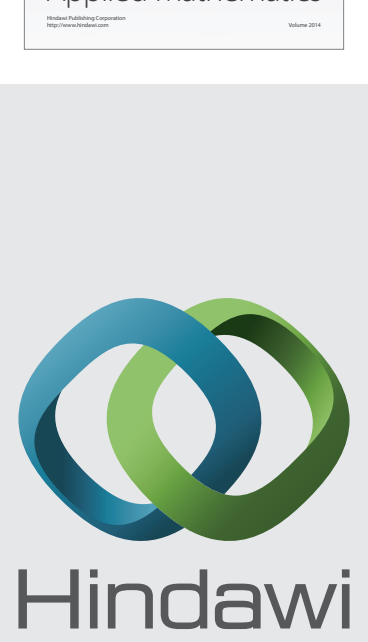

Submit your manuscripts at http://www.hindawi.com
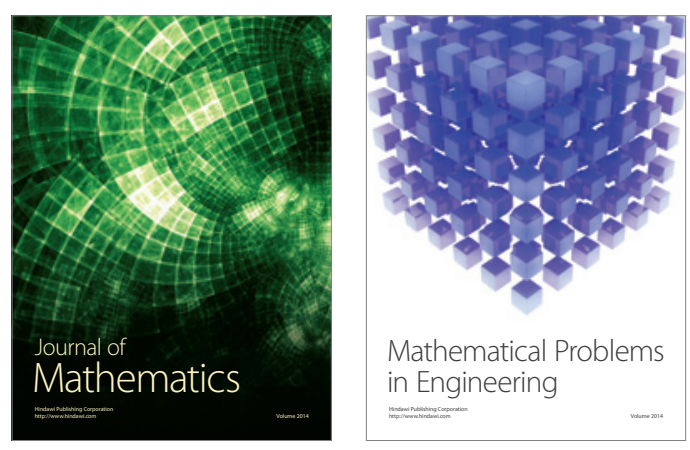

Mathematical Problems in Engineering
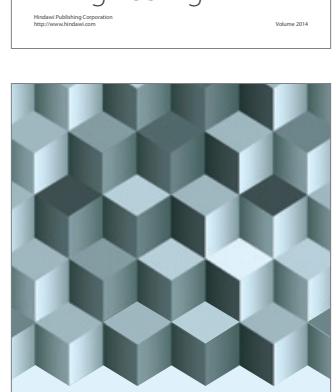

Journal of

Function Spaces
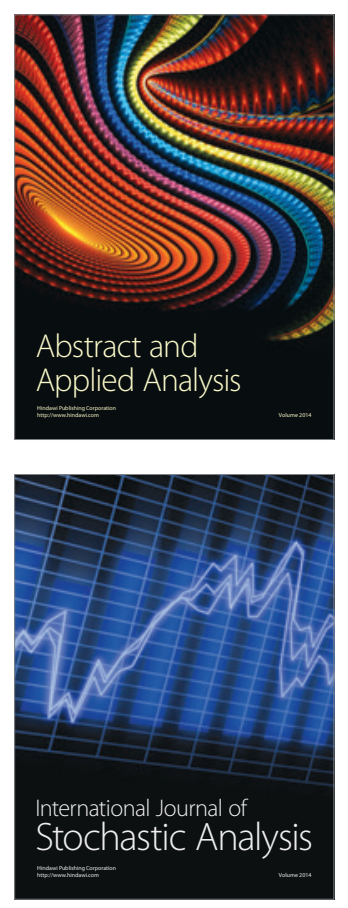

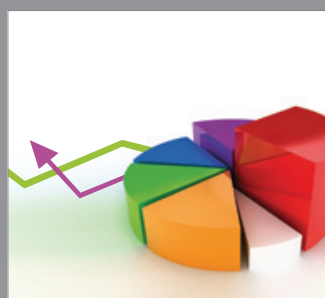

ournal of

Probability and Statistics

Promensencen
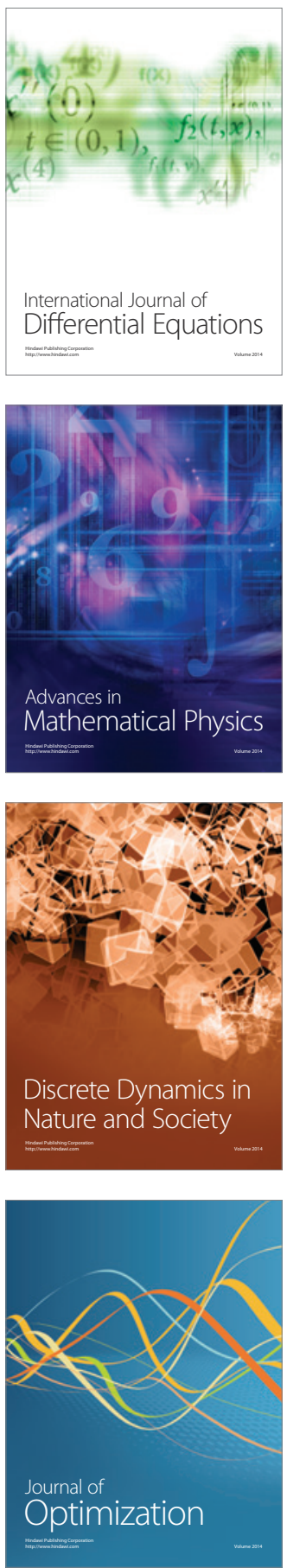